Pacific Journal of Mathematic 


\title{
ALTERNIERENDE PRODUKTE IN FREIEN GRUPPEN
}

\author{
GERHARD RosenberGER
}

1. Einleitung. A. In [7] and [8] untersucht H. Zieschang das Problem, wann zwei alternierende Produkte in freien Gruppen verwandt sind. Bei Anwendungen und konkreten Problemen tritt aber oft die allgemeinere Frage auf:

Sei $G$ freie Gruppe vom Rang $n \geqq 1$ mit den freien Erzeugenden $a_{1}, \cdots, a_{n}$ und

$$
P\left(a_{1}, \cdots, a_{n}\right)=a_{1}^{\alpha_{1}} \cdots a_{p}^{\alpha}\left[a_{p+1}, a_{p+2}\right] \cdots\left[a_{n-1}, a_{n}\right] \in G
$$

ein alternierendes Produkt in $G$. Sei $X$ Untergruppe von $G$ vom Rang $m \geqq 1$ und $P^{\alpha}\left(a_{1}, \cdots, a_{n}\right) \in X$ für ein $\alpha \geqq 1$. Wie läßt sich $X$ durch $P\left(a_{1}, \cdots, a_{n}\right)$ beschreiben? Diese Frage wurde beispielsweise schon zum Teil beantwortet in [5] (der Fall $p=n$ ) und [7] (der Fall $p=0$ ). Hier geben wir eine vollständige Antwort auf diese Frage.

Im zweiten Teil der Arbeit finden die Ergebnisse zu dieser Frage Anwendungen bei der Behandlung gewisser Untergruppenprobleme von freien Gruppen und bei der Beschreibung von Erzeugendensystemen einer Klasse von Gruppen. Insbesondere lösen wir für eine Klasse von Gruppen mit einer definierenden Relation das Isomorphieproblem in dem Sinn, daß wir in endlich vielen Schritten entscheiden können, ob eine beliebige Gruppe mit einer definierenden Relation $\mathrm{zu}$ einer Gruppe dieser Klasse isomorph ist oder nicht.

B. Diese Arbeit verwendet die Terminologie und Bezeichnungsweise von [4] und [9]. Es bedeute: $[a, b]=a b a^{-1} b^{-1}$ den Kommutator von $a, b \in G$ (G Gruppe). $\langle\cdots ; \cdots\rangle$ die Gruppenbeschreibung durch Erzeugende und Relationen. $\left(\beta_{1}, \cdots, \beta_{k}\right)$ den größten gemeinsamen Teiler von $\beta_{1}, \cdots, \beta_{k} \in N(k \geqq 1)$.

2. Über Gleichungen in freien Gruppen. Sei $G=\left\langle a_{1}, \cdots, a_{n} ;\right\rangle$ $(n \geqq 1)$ freie Gruppe vom Rang $n$ mit den freien Erzeugenden $a_{1}, \cdots, a_{n}$ und

$$
P\left(a_{1}, \cdots, a_{n}\right)=a_{1}^{\kappa_{1}} \cdots a_{p}^{\alpha_{p}}\left[a_{p+1}, a_{p+2}\right] \cdots\left[a_{n-1}, a_{n}\right] \in G
$$

ein alternierendes Produkt in $G$ mit $0 \leqq p \leqq n, \alpha_{i} \geqq 1$ für $i=1, \cdots, p$. Sei $\left\{x_{1}, \cdots, x_{m}\right\} \subset G(m \geqq 1)$ und $X$ die von den $x_{1}, \cdots, x_{m}$ erzeugte Untergruppe von $G$. Sei $y^{-1} P^{\alpha}\left(a_{1}, \cdots, a_{n}\right) y \in X$ für ein $\alpha \neq 0$ und $y \in G$. 
SATz 1. Es tritt einer der folgenden Fälle ein:

(a) Es gibt einen freien Übergang von $\left\{x_{1}, \cdots, x_{m}\right\}$ zu einem System $\left\{y_{1}, \cdots, y_{m}\right\}$ mit $y_{1}=z P^{\beta}\left(a_{1}, \cdots, a_{n}\right) z^{-1}, \beta>0$ und $z \in G$, und $\beta$ ist die kleinste positive Zahl, für die eine Beziehung $y^{-1} P^{\beta}\left(a_{1}, \cdots, a_{n}\right) y \in X$ für ein $y \in G$ gilt.

(b) Es ist $m \geqq n$, und es gibt einen freien Übergang von $\left\{x_{1}, \cdots, x_{m}\right\} z u$ einem System $\left\{y_{1}, \cdots, y_{m}\right\}$ mit $y_{i}=z a_{i}^{\gamma} z^{-1}, 1 \leqq \gamma_{i}<\alpha_{i}$. $\gamma_{i} \mid \alpha_{i}(i=1, \cdots, p), y_{j}=z a_{j} z^{-1}(j=p+1, \cdots, n)$ und $z \in G$.

Beweis. Es trete nicht der Fall (a) ein. Wir können annehmen, daß kein zu $\left\{x_{1}, \cdots, x_{m}\right\}$ frei äquivalentes System $\left\{y_{1}, \cdots, y_{q}\right\}$ ein echtes Teilsystem $\left\{y_{1}, \cdots, y_{q}\right\}$ besitzt mit $q<m$ und $y^{-1} P^{\alpha}\left(a_{1}, \cdots, a_{m}\right) y \in\left\langle y_{1}, \cdots, y_{q}\right\rangle$ für ein $\alpha \neq 0$ und $y \in G$. Wir dürfen also im folgenden voraussetzen: $m$ ist in diesem Sinn minimal mit dieser Eigenschaft, und $\left\{x_{1}, \cdots, x_{m}\right\}$ ist insbesondere schon ein freies Erzeugendensystem von $X$.

Im folgenden sei $\alpha$ die kleinste positive Zahl, für die eine Beziehung $y^{-1} P^{\alpha}\left(a_{1}, \cdots, a_{n}\right) y \in X$ für ein $y \in G$ gilt. Wir dürfen $y=1$ annehmen-eventuell nach Ersetzen von $x_{i}$ durch $y x_{i} y^{-t}$.

In $G$ sei die freie Länge $L$ und eine geeignete lexikographische Ordnung relativ zu den Erzeugenden $a_{1}, \cdots, a_{n}$ eingeführt. Wenden wir die klassische Nielsensche Kürzungsmethode auf $\left\{x_{1}, \cdots, x_{m}\right\}$ an, so erhalten wir schließlich ein System, das der Nielsenschen Eigenschaft bzgl. $L$ genügt, d.h. in dem kein Element und kein Inverses eines Elementes von einem anderen mehr als die Hälfte kürzt, keine zwei ein anderes ganz kürzen (vgl. [10, S. I. 12]). Das neue System erzeugt dieselbe Untergruppe von $G$ wie $\left\{x_{1}, \cdots, x_{m}\right\}$. Wir können nun annehmen, daß schon $\left\{x_{1}, \cdots, x_{m}\right\}$ die Nielsensche Eigenschaft bzgl. $L$ hat. Wir schreiben nun jedes $x_{1}^{\varsigma}, \varepsilon= \pm 1$; als frei gekürzten Wort in $a_{1}, \cdots, a_{n}$. Dann bleibt in jedem frei gekürzten Produkt in $x_{1}, \cdots, x_{m}$ für jedes $x_{i}^{\varepsilon}, \varepsilon= \pm 1$, mindestens ein Symbol ungeändert an der Stelle erhalten, an der $x_{i}^{\varepsilon}$ auftrat, wenn man für die $x_{1}, \cdots, x_{m}$ ihre frei gekürzten Worte in $a_{1}, \cdots, a_{n}$ einsetzt und relativ $\mathrm{zu} a_{1}, \cdots, a_{n}$ frei kürzt. Für jedes $x_{i}$ zeichnen wir eine solche standhafte Stelle aus und nehmen für $x_{i}^{-1}$ die entsprechende Stelle mit dem inversen Zeichen. $\left\{x_{1}, \cdots, x_{m}\right\}$ genügt einer Gleichung

$$
\prod_{j=1}^{t_{1}} x_{\nu_{j}}^{\varepsilon_{j}}=W\left(x_{1}, \cdots, x_{m}\right)=P^{\alpha}\left(a_{1}, \cdots, a_{n}\right),
$$

$\varepsilon_{j}= \pm 1, \varepsilon_{j}=\varepsilon_{j+1}$ falls $\nu_{j}=\nu_{j+1}$.

Beh. (2.2). Es ist $\alpha=1$.

Beweis von (2.2). Angenommen $\alpha \geqq 2$. Da der Fall (a) nicht eintritt, ist $W\left(x_{1}, \cdots, x_{m}\right)$ kein primitives Element in $\left\langle x_{1}, \cdots, x_{m}\right\rangle$. 
Nach [1] ist $W\left(x_{1}, \cdots, x_{m}\right)$ eine echte Potenz in $X$. Das ist ein Widerspruch zur Minimalität von $\alpha$. Also ist $\alpha=1$.

Sei nun $\alpha=1$. Für $p=n$ folgt die Behauptung aus Satz 1 von [5]. Für $p=0$ folgt die Behauptung aus Satz 2 [5]. Sei nun $1 \leqq p<n$.

Es gebe nun in (2.1) zwei verschiedene Indizes $j, i\left(1 \leqq j<i \leqq t_{1}\right)$ mit $\nu_{j}=\nu_{i}=k, \nu_{h} \neq k$ für $j<h<i$ und $\varepsilon_{j}=\varepsilon_{i}$. Sei ohne Einschränkung $\varepsilon_{j}=\varepsilon_{\imath}=1$. Wir wollen zeigen, da $j=i-1$ und $x_{k}$ zu einer Potenz von einem $a_{\nu}(1 \leqq \nu \leqq p)$ konjugiert ist. Sei $a_{\lambda}^{\varepsilon}, \varepsilon= \pm 1$, eine standhafte Stelle von $x_{k}$. Es bleibt $a_{\lambda}^{\varepsilon}$ beide Male stehen. Daher tritt in (2.1) nicht $x_{k}^{-1}$ auf, denn das standhafte Zeichen von $x_{k}^{-1}$ ist $a_{\lambda}^{-\varepsilon}$, und in (2.1) kann das standhafte Zeichen $a_{\lambda}^{-\varepsilon}$ nicht auftreten, wenn das standhafte Zeichen $a_{\lambda}^{\varepsilon}$ zweimal auftritt. Insbesondere ist $1 \leqq \lambda \leqq p$ und $\varepsilon=1$. Es ist $x_{k}=u a_{\lambda}^{\beta} v$, wobei das frei gekürzte Wort von $u$ bzw. $v$ in $a_{1}, \cdots, a_{n}$ nicht mit Potenzen von $a_{\lambda}$ endet bzw. beginnt. Weiter ist

$$
\begin{aligned}
x_{k}( & \left.\prod_{\mu=j+1}^{i-1} x_{\nu_{\mu}^{\mu}}^{\varepsilon_{\mu}}\right) x_{k}=u a_{\lambda}^{\beta} v\left(\prod_{\mu=j+1}^{i-1} x_{\nu_{\mu}}^{\varepsilon_{\mu}}\right) u a_{\lambda}^{\beta} v \\
& =u a_{\lambda}^{\beta^{\prime}}\left(a_{\lambda}^{\alpha_{\lambda}} \cdots a_{p}^{\alpha}\left[a_{p+1}, a_{p+2}\right] \cdots\left[a_{n-1}, a_{n}\right] a_{\llcorner}^{\alpha_{1}} \cdots a_{\lambda-1}^{\alpha_{\lambda}-1}\right)^{\delta} \cdot a_{\lambda}^{\beta^{\prime \prime}} \cdot v, \\
& \delta \geqq 0 .
\end{aligned}
$$

Da in $P\left(a_{1}, \cdots, a_{n}\right)$ zwischen zwei Zeichen $a_{\lambda}$ keine anderen $a_{\nu}$ auftreten, ist $\delta=0$. Dann ist $j=i-1$ und $x_{k}$ ist zu einer Potenz von $a_{\lambda}$ konjugiert (Wegen der Minimalität von $m$, und da (a) nicht eintritt).

Wir haben also:

Tritt in (2.1) ein $x_{j}$ mindestens zweimal mit dem gleichen Exponenten auf, so ist dieses $x_{j}$ zu einer Potenz von einem $a_{i}(1 \leqq i \leqq p)$ konjugiert.

Da der Fall (a) nicht eintritt, erhalten wir gleichzeitig: Jedes $x_{j}$, das in (2.1) vorkommt, tritt dort entweder mindestens zweimal mit dem gleichen Exponenten, d.h. ist zu einer Potenz von einem $a_{i}(1 \leqq i \leqq p)$ konjugiert, oder genau einmal mit dem Exponenten +1 und genau einmal mit dem Exponenten -1 auf.

Sei $r(0 \leqq r \leqq m)$ die Zahl der $x_{j}$, die in (2.1) mindestens zweimal mit dem gleichen Exponenten auftreten, und sei $s(0 \leqq s \leqq m)$ die Zahl der $x_{j}$, die in (2.1) genau einmal mit dem Exponenten +1 und genau einmal mit dem Exponenten -1 auftreten.

Indem wir unwesentliche und überflüssige Elemente entfernen, können wir anehmen, daß jedes $x_{j}$ in (2.1) auftritt, und wir bezeichnen das neue System flüchtig mit den gleichen Symbolen. Damit ist $1 \leqq r+s=m$ (Die nachfolgenden Überlegungengen zeigen $m=n$ ). 
Beh. (2.3). Es ist $r=p$.

Beweis von (2.3). Angenommen $r<p$. Dann gibt es ein $a_{\lambda}(1 \leqq \lambda \leqq p)$, für das kein $x_{\nu}$ zu einer Potenz von diesem $a_{\lambda}$ konjugiert ist, etwa $a_{1}$. Dann liegt in der abelsch-gemachten Gruppe ein $a_{1}^{\gamma}, \gamma \neq 0$, in der von den $a_{2}, \cdots, a_{n}$ erzeugten abelschen Gruppe. Das ist aber ein Widerspruch dazu, daß die abelsch-gemachte Gruppe freie abelsche Gruppe vom Rang $n$ ist.

Also ist $r \geqq p$. Es ist auch $r \leqq p$, denn für $r>p$ sind zwei verschiedene $x_{j}$ zu einer Potenz desselben $a_{\lambda}(1 \leqq \lambda \leqq p)$ konjugiert, und das steht im Widerspruch dazu, daß das System $\left\{x_{1}, \cdots, x_{m}\right\}$ Nielsensche Eigenschaft hat und (2.1) genügt und jedes $x_{j}$ in (2.1) auftritt. Also ist $r=p$.

Aus der Nielsenschen Eigenschaft von $\left\{x_{1}, \cdots, x_{m}\right\}$ folgt nun, $\mathrm{da} B$ wir-eventuell nach einem geeigneten freien Übergang und Umnummerierung-

$$
x_{i}=a_{i}^{i}, 1 \leqq \gamma_{i}<\alpha_{i}, \quad \gamma_{i} \mid \alpha_{i} \quad \text { für } \quad i=1, \cdots, p
$$

annehmen können.

Damit haben wir Satz 1 auf die folgende Situation zurückgeführt: $\left\{x_{p+1}, \cdots, x_{m}\right\}$ genügt einer Gleichung

$$
\prod_{j=1}^{t_{2}} x_{\nu_{j}}^{\varepsilon_{j}}=\left[a_{p+1}, a_{p+2}\right] \cdots\left[a_{n-1}, a_{n}\right], \quad \varepsilon_{j}= \pm 1,
$$

$\varepsilon_{j}=\varepsilon_{j+1}$ falls $\nu_{j}=\nu_{j+1}$, in der jedes $x_{j}(p+1 \leqq j \leqq m)$ genau einmal mit dem Exponenten +1 und genau einmal mit dem Exponenten -1 auftritt (es können noch Erzeugende $a_{i}(1 \leqq i \leqq p)$ in den Worten $x_{j}(p+1 \leqq j \leqq m)$ auftreten). Es ist $m \leqq n$, da sonst in (2.3) die Zahl der standhaften Zeichen größer als $2(n-p)$ wäre, d.h.

$$
L\left(\left[a_{p+1}, a_{p+2}\right] \cdots\left[a_{n-1}, a_{n}\right]\right)=2(n-p) \geqq t_{2}-1 \geqq 2(n-p)+1
$$

wäre. Andererseits ist $m \geqq n$ (vgl. [5] und [2]).

Also ist $m=n=r+s=p+s$.

Analog wie beim Beweis von Satz V. 2 von [10; pp. V. 6 und V. 7] zeigt man nun aber: Es gibt einen freien Übergang von

$$
\left\{a_{1}^{\gamma_{1}}, \cdots, a_{p}^{\gamma}, x_{p+1}, \cdots, x_{m}\right\} \text { zu }\left\{a_{1}^{\gamma}, \cdots, a_{p}^{\gamma p}, a_{p+1}, \cdots, a_{n}\right\} .
$$

Korollar 1. Sei $\left(\alpha_{1}, \cdots, \alpha_{p}\right) \geqq 2$. Ist $\varphi$ ein Endomorphismus von $G, \operatorname{der} P\left(a_{1}, \cdots, a_{n}\right)$ festläßt, so ist $\varphi$ ein Automorphismus von $G$.

Beweis. Wir setzen $y_{i}=\varphi\left(a_{i}\right)$ für $i=1, \cdots, n$ und betrachten die von $y_{1}, \cdots, y_{n}$ erzeugte Untergruppe $Y$ von $G$. In $Y$ gilt

$$
P\left(y_{1}, \cdots, y_{n}\right)=P\left(a_{1}, \cdots, a_{n}\right) \text {. }
$$


Analog wie bei Lemma 1 von [5] ergibt sich wegen $\left(\alpha_{1}, \cdots, \alpha_{p}\right) \geqq 2$, $\mathrm{da} \beta$ es keinen freien Übergang von $\left\{y_{1}, \cdots, y_{n}\right\}$ zu einem System $\left\{z_{1}, \cdots, z_{n}\right\}$ mit $z_{1}=P\left(a_{1}, \cdots, a_{n}\right)$ gibt. Nun folgt die Behauptung aus Satz 1 .

\section{Anwendungen.}

SATZ 2. Sei $G=\left\langle a_{1}, \cdots, a_{n} ;\right\rangle(n \geqq 1)$ freie Gruppe vom Rang $n$ mit den freien Erzeugenden $a_{1}, \cdots, a_{n}$. Sei $\left\{x_{1}, \cdots, x_{m}\right\} \subset G(m \geqq 1)$ und $X$ die von $x_{1}, \cdots, x_{m}$ erzeugte Untergruppe von $G$. Es gebe $u_{1}, \cdots, u_{k} \in X$ mit

$$
\begin{gathered}
u_{1}^{\beta_{1}} \cdots u_{k}^{\beta_{k}}=a_{1}^{\alpha_{1}} \cdots a_{p}^{\alpha}\left[a_{p+1}, a_{p+2}\right] \cdots\left[a_{n-1}, a_{n}\right], \\
0 \leqq p \leqq n, \alpha_{i} \geqq 1(i=1, \cdots, p), 2 \leqq \beta_{j}(j=1, \cdots, k) \text { und }\left(\beta_{1}, \cdots, \beta_{k}\right) \geqq 2 .
\end{gathered}
$$

Dann gilt.

(a) Es ist $m \geqq n$, und es gibt einen freien Übergang von $\left\{x_{1}, \cdots, x_{m}\right\}$ zu einem System $\left\{y_{1}, \cdots, y_{m}\right\}$ mit $y_{i}=a_{i}^{\gamma_{i}}, 1 \leqq \gamma_{i}<\alpha_{i}$, $\gamma_{i} \mid \alpha_{i}(i=1, \cdots, p), y_{j}=a_{j}(j=p+1, \cdots, n)$.

(b) Ist $p=n$, so ist $k \geqq n$.

(c) Ist $p<n$, so ist $k \geqq n+1$.

Beweis. $X$ ist als Untergruppe einer freien Gruppe vom Rang $n$ auch freie Gruppe. Es ist $q=\operatorname{Rang} X \leqq m$, da $X$ von $x_{1}, \cdots, x_{m}$ erzeugt wird. Sei $\left\{y_{1}, \cdots, y_{q}\right\}$ ein freies Erzeugendensystem von $X$. Nach Lemma 1 von [5] gibt es keinen freien Übergang von $\left\{y_{1}, \cdots, y_{q}\right\}$ zu einem System $\left\{z_{1}, \cdots, z_{q}\right\}$ mit $z_{1}=a_{1}^{\alpha_{1}} \cdots a_{p}^{\alpha}\left[a_{p+1}, a_{p+2}\right] \cdots\left[a_{n-1}, a_{n}\right]$. Damit gilt nach Satz 1:

Es ist $q \geqq n$, und es gibt einen freien Übergang von $\left\{y_{1}, \cdots, y_{q}\right\}$ zu einem System $\left\{z_{1}, \cdots, z_{q}\right\}$ mit $z_{i}=\alpha_{i}^{r_{i}}, 1 \leqq \gamma_{i}<\alpha_{i}, \gamma_{i} \mid \alpha_{i}(i=1, \cdots, p)$, $z_{j}=a_{j}(j=p+1, \cdots, n)$.

Insbesondere ist $k \geqq n$. $\mathrm{Zu}$ zeigen ist noch: Für $p<n$ ist $k \geqq n+1$.

Sei nun $p<n$. Angenommen $k=n$.

Dann gibt es einen freien Übergang von $\left\{u_{1}, \cdots, u_{k}\right\}$ zu einem System $\left\{a_{1}^{\gamma_{1}}, \cdots, a_{p}^{\gamma_{p}}, a_{p+1}, \cdots, a_{n}\right\}$ mit $1 \leqq \gamma_{i}<\alpha_{i}, \gamma_{i} \mid \alpha_{i}(i=1, \cdots, p)$.

Wir können also ohne Einschränkung $p=0$ annehmen (andernfalls fügen wir die Relationen $a_{1}=\cdots=a_{p}=1$ hinzu).

Sei nun $p=0$. Es ist $u_{1}^{\beta_{1}} \cdots u_{k}^{\beta_{k}}=1$ in der abelsch-gemachten Gruppe, und das ist ein Widerspruch dazu, daß diese freie abelsche Gruppe vom Rang $n=k$ ist. Also ist $k \geqq n+1$.

KorollaR 2. Es mögen die Voraussetzungen von Satz 2 gelten. Ferner sei $\alpha_{i}$ Primzahl für $i=1, \cdots, p$. Dann gilt: 
Ist $m=n$, so ist $\left\{x_{1}, \cdots, x_{m}\right\}$ freies Erzeugendensystem von $G$. Ist $m \geqq n$, so ist $X=G$.

BEMERKung. Wir haben damit eine einfache notwendige und hinreichende Bedingung dafür, wann ein System $\left\{x_{1}, \cdots, x_{n}\right\} \subset G$ ein freies Erzeugendensystem der freien Gruppe $G$ vom Rang $n$ ist.

Korollar 3. Seien $G$ und $X$ wie in Satz 2. Es gebe $u_{1}, \cdots, u_{k} \in X$ mit $u_{1}^{\beta_{1}} \cdots u_{q}^{\beta_{q}}\left[u_{q+1}, u_{q+2}\right] \cdots\left[u_{k-1}, u_{k}\right]=a_{1}^{\alpha_{1}} \cdots a_{p}^{\alpha_{p}}\left[a_{p+1}, a_{p+2}\right] \cdots\left[a_{n-1}, a_{n}\right]$ $0 \leqq p \leqq n, \alpha_{i} \geqq 1(i=1, \cdots, p), 0 \leqq q \leqq k, 2 \leqq \beta_{j}(j=1, \cdots, q)$ und $\left(\beta_{1}, \cdots, \beta_{q}\right) \geqq 2$.

Dann ist $m \geqq n$, insbesondere ist $k \geqq n$, und es gibt einen freien Übergang von $\left\{x_{1}, \cdots, x_{m}\right\}$ zu einem System $\left\{y_{1}, \cdots, y_{m}\right\}$ mit $y_{\imath}=a_{i}^{\gamma_{2}}$, $1 \leqq \gamma_{i}<\alpha_{i}, \gamma_{i} \mid \alpha_{i}(i=1, \cdots, p), y_{j}=a_{j}(j=p+1, \cdots, n)$.

Ist $\alpha_{i}$ Primzahl für $i=1, \cdots, p$, so ist sogar $X=G$.

Indem wir analog wie beim Beweis von Satz 13 und Satz 24 von [4] vorgehen, erhalten wir:

SATZ 3. Sei

$$
\begin{aligned}
K & =\left\langle s_{1}, \cdots, s_{m}, a_{1}, \cdots, a_{n}\right| s_{1}^{\gamma_{1}}=\cdots=s_{m}^{\gamma_{m}} \\
& \left.=s_{1} \cdots s_{m}\left(a_{1}^{\alpha_{1}} \cdots a_{p}^{\alpha_{p}}\left[a_{p+1}, a_{p+2}\right] \cdots\left[a_{n-1}, a_{n}\right]\right)^{\gamma}=1\right\rangle
\end{aligned}
$$

mit $\gamma_{i} \geqq 2, \alpha_{j} \geqq 2, \gamma \geqq 1, m \geqq 2, n \geqq 2,0 \leqq p \leqq n . \quad$ Ist $\left\{x_{1}, \cdots, x_{n+m-1}\right\}$ ein Erzeugendensystem von $K$, so gibt es einen freien Übergang von $\left\{x_{1}, \cdots, x_{n+m-1}\right\}$ zu einem System $\left\{s_{\nu_{1}}^{\beta_{1}}, \cdots, s_{\nu_{m-1}}^{\beta_{m-1}}, a_{1}, \cdots, a_{n}\right\}$ mit $\nu_{i} \in\{1, \cdots, m\}, \quad \nu_{1}<\cdots<\nu_{m+1} \quad($ falls $m>2) \quad$ und $1 \leqq \beta_{i}<\alpha_{\nu_{\imath}}$, $\left(\beta_{i}, \alpha_{\nu_{i}}\right)=1$.

Indem wir mit ähnlichen, verfeinerten Methoden wie in [6] vorgehen, erhalten wir:

SATZ 4. Sei $H=\left\langle a_{1}, \cdots, a_{n}, b_{1}, \cdots, b_{m}\right| W\left(a_{1}, \cdots, a_{n}\right) V\left(b_{1}, \cdots, b_{m}\right)=$ 1) mit $W\left(a_{1}, \cdots, a_{n}\right)=\left(a_{1}^{\alpha_{1}} \cdots a_{p}^{\alpha_{p}}\left[a_{p+1}, a_{p+2}\right] \cdots\left[a_{n-1}, a_{n}\right]\right)^{\gamma}, V\left(b_{1}, \cdots, b_{m}\right)=$ $\left(b_{1}^{\beta_{1}} \cdots b_{q}^{\beta_{q}}\left[b_{q+1}, b_{q+2}\right] \cdots\left[b_{m-1}, b_{m}\right]\right)^{\delta}, \alpha_{i} \geqq 2, \beta_{j} \geqq 2, \gamma \geqq 2, \delta \geqq 2, n \geqq 2, m \geqq 2$, $0 \leqq p \leqq n, 0 \leqq q \leqq m$ oder $\alpha_{i} \geqq 2, \gamma \geqq 2, n \geqq 2,0 \leqq p \leqq n, m \geqq 1$, $\delta=1, q=0$. Ist $\left\{x_{1}, \cdots, x_{n+m}\right\}$ ein Erzeugendensystem von $H$, so gibt es einen freien Übergang von $\left\{x_{1}, \cdots, x_{n+m}\right\}$ zu $\left\{a_{1}, \cdots, a_{n}\right.$, $\left.b_{1}, \cdots, b_{m}\right\}$.

Die Methode von Whitehead liefert nun (vgl. [3, Satz 1] oder [6]):

Korollar 4. Sei $H$ wie in Satz 4 gegeben. Dann ist das 
Isomorphieproblem für $H$ lösbar in dem Sinn, daß wir in endlich vielen Schritten entscheiden können, ob eine gegebene Gruppe mit einer definierenden Relation $z u H$ isomorph ist oder nicht.

\section{Ähnlich wie in [3, Satz 2 und Satz 3] folgt nun:}

Korollar 5. Sei $H$ wie in Satz 4 gegeben. Dann gilt:

(1) $H$ ist eine Hopfsche Gruppe, d.h. jeder Endomorphismus von $H$ auf $H$ ist ein Automorphismus von $H$.

(2) Jeder Automorphismus von $H$ wird von einem Automorphismus der freien Gruppe von Rang $n+q$ induziert.

(3) Die Automorphismengruppe von $H$ ist endlich erzeugt.

Für $\delta=1$ und $q \geqq 1$ erhalten wir:

SATZ 5. Sei $H=\left\langle a_{1}, \cdots, a_{n}, b_{1}, \cdots, b_{m}\right| W\left(a_{1}, \cdots, a_{n}\right) V\left(b_{1}, \cdots, b_{m}\right)=$ 1) mit $W\left(a_{1}, \cdots, a_{n}\right)=\left(a_{1}^{\alpha_{1}} \cdots a_{p}^{\alpha_{p}}\left[a_{p+1}, a_{p+2}\right] \cdots\left[a_{n-1}, a_{n}\right]\right)^{\gamma}, V\left(b_{1}, \cdots, b_{m}\right)=$ $b_{1}^{\beta_{1}} \cdots b_{q}^{\beta_{q}}\left[b_{q+1}, b_{q+2}\right] \cdots\left[b_{m-1}, b_{m}\right], \alpha_{i} \geqq 2, \beta_{j} \geqq 2, \gamma \geqq 2, n \geqq 2, m \geqq 1$, $0 \leqq p \leqq n, 1 \leqq q \leqq m$. Ist $\left\{x_{1}, \cdots, x_{n+m}\right\}$ ein Erzeugendensystem von $H$, so gibt es einen freien Übergang von $\left\{x_{1}, \cdots, x_{n+m}\right\}$ zu einem System $\left\{a_{1}, \cdots, a_{n}, b_{1}, \cdots, b_{i-1}, b_{i}^{r_{i}}, b_{i+1}, \cdots, b_{q}, b_{q+1}, \cdots, b_{m}\right\}$ für ein $i$ $(1 \leqq i \leqq q)$ mit $\left(\gamma_{i}, \beta_{i}\right)=1$ und $1 \leqq \gamma_{i} \leqq \frac{1}{2} \beta_{i}$.

Für $\delta=1$ und $\gamma=1$ gilt Satz 3 von [6].

\section{LITERATUR}

1. G. Baumslag, Residual nilpotence and relations in free groups, J. Algebra, 2 (1965), 271-282.

2. C. C. Edmunds, Some properties of quadratic words in free groups, Proc. Amer. Math. Soc., 50 (1975), 20-22.

3. S. J. Pride, The isomorphism problem for two-generator one-relator groups with torsion is solvable, (Preprint).

4. G. Rosenberger, Zum Rang- und Isomorphieproblem für freie Produkte mit Amalgam. Habilitationsschrift, Hamburg 1974.

5. - Produkte von Potenzen und Kommutatoren in freien Gruppen, J. of Algebra, 53 (1978), 416-422.

6. Z Zum Isomorphieproblem für Gruppen mit einer definierenden Relation, Illinois J. Math., 20 (1976), 614-621.

7. H. Zieschang, Alternierende Produkte in freien Gruppen, Abh. Math. Sem. Univ. Hamburg, 27 (1964), 13-31.

8. - Alternierende Produkte in freien Gruppen II, Abh. Math. Sem. Univ. Hamburg, 28 (1965), 219-233.

9. — Über die Nielsensche Kürzungsmethode in freien Produkten mit Amalgam, Inventiones Math., 10 (1970), 4-37. 
10. H. Zieschang, E. Vogt, and H.-D. Coldewey, Flächen und ebene diskontinuierliche Gruppen, Lecture notes in Math., 122, Springer 1970.

Received December 20, 1976.

Abteilung Mathematik

DER UNIVERSITÄT DORTMUND

POSTFACH 500500

4600 DoRTMund 50 


\section{PACIFIC JOURNAL OF MATHEMATICS}

\section{EDITORS}

RICHARD ARENS (Managing Editor)

University of California

Los Angeles, California 90024

C. W. CurTis

University of Oregon

Eugene, OR 97403

C. C. MOORE

University of California

Berkeley, CA 94720
J. DUGUNDJI

Department of Mathematics University of Southern California Los Angeles, California 90007

R. FinN AND J. Milgram Stanford University Stanford, California 94305

\section{ASSOCIATE EDITORS}

E. F. BECKENBACH

B. H. NeumanN

F. WOLF

K. YoSHIDA

\section{SUPPORTING INSTITUTIONS}

UNIVERSITY OF BRITISH COLUMBIA CALIFORNIA INSTITUTE OF TECHNOLOGY UNIVERSITY OF CALIFORNIA MONTANA STATE UNIVERSITY UNIVERSITY OF NEVADA, RENO NEW MEXICO STATE UNIVERSITY OREGON STATE UNIVERSITY UNIVERSITY OF OREGON
UNIVERSITY OF SOUTHERN CALIFORNIA

STANFORD UNIVERSITY

UNIVERSITY OF HAWAII

UNIVERSITY OF TOKYO

UNIVERSITY OF UTAH

WASHINGTON STATE UNIVERSITY

UNIVERSITY OF WASHINGTON 


\section{Pacific Journal of Mathematics \\ Vol. 78, No. $1 \quad$ March, 1978}

Simeon M. Berman, A class of isotropic distributions in $\mathbf{R}^{n}$ and their

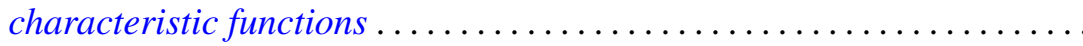

Ezra Brown and Charles John Parry, The 2-class group of biquadratic fields.

II ........................................ 11

Thomas E. Cecil and Patrick J. Ryan, Focal sets of submanifolds ....... 27

Joseph A. Cima and James Warren Roberts, Denting points in $B^{p} \ldots \ldots \ldots 41$

Thomas W. Cusick, Integer multiples of periodic continued fractions . . . . . 47

Robert D. Davis, The factors of the ramification sequence of a class of

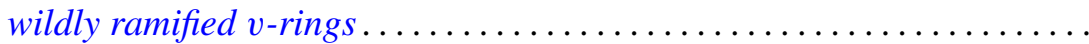

Robert Martin Ephraim, Multiplicative linear functionals of Stein

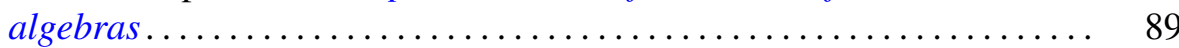

Philip Joel Feinsilver, Operator calculus . .................... 95

David Andrew Gay and William Yslas Vélez, On the degree of the splitting field of an irreducible binomial ..........................

Robert William Gilmer, Jr. and William James Heinzer, On the divisors of

monic polynomials over a commutative ring ..................

Robert E. Hartwig, Schur's theorem and the Drazin inverse .............

Hugh M. Hilden, Embeddings and branched covering spaces for three and four dimensional manifolds ............................

Carlos Moreno, The Petersson inner product and the residue of an Euler product. ...

Christopher Lloyd Morgan, On relations for representations of finite groups....

Ira J. Papick, Finite type extensions and coherence

$\mathrm{R}$. Michael Range, The Carathéodory metric and holomorphic maps on a class of weakly pseudoconvex domains ................

Donald Michael Redmond, Mean value theorems for a class of Dirichlet series

Daniel Reich, Partitioning integers using a finitely generated semigroup ...

Georg Johann Rieger, Remark on a paper of Stux concerning squarefree

numbers in non-linear sequences

Gerhard Rosenberger, Alternierende Produkte in freien Gruppen ..

Ryōtarō Satō, Contraction semigroups in Lebesgue space

Tord Sjödin, Capacities of compact sets in linear subspaces of $\mathbf{R}^{n}$

Robert Jeffrey Zimmer, Uniform subgroups and ergodic actions of exponential Lie groups......................... 